\title{
Comparison of CT, PET, and PET/CT for Staging of Patients with Indolent Non-Hodgkin's Lymphoma
}

\author{
Barbara J. Fueger, ${ }^{1}$ Kristen Yeom, ${ }^{2}$ Johannes Czernin, ${ }^{1}$ James W. Sayre, ${ }^{2}$ \\ Michael E. Phelps, ${ }^{1}$ Martin S. Allen-Auerbach ${ }^{1,3}$ \\ ${ }^{1}$ Department of Molecular and Medical Pharmacology, David Geffen School of Medicine, UCLA, Los Angeles, CA 90095, USA \\ ${ }^{2}$ Department of Radiology, David Geffen School of Medicine, UCLA, Los Angeles, CA 90095, USA \\ ${ }^{3}$ Department of Nuclear Medicine, UCLA School of Medicine, AR-243 CHS, 10833 Le Conte Avenue, Los Angeles, CA 90095-6942, USA
}

\begin{abstract}
Purpose: The aim was to investigate the potential impact of positron emission tomography (PET)/computed tomography (CT) as compared to PET and CT on the staging of patients with indolent lymphoma.

Procedures: PET/CTs from 45 patients with indolent lymphoma undergoing staging or restaging were studied. Clinical follow-up, additional imaging, and histology served as the gold standard. Results: PET/CT correctly diagnosed 92 nodal regions as positive for lymphomatous involvement and 458 as disease free vs 68 and 449 for PET and 64 and 459 for CT, respectively. The respective sensitivities, specificities, and accuracies were $99 \%, 100 \%$, and $99.8 \%$ for PET/CT, $68 \%, 97.5 \%$, and $92.2 \%$ for PET, and $70 \%, 100 \%$, and $94.7 \%$ for CT. PET/CT performed significantly better than PET ( $p<0.001$ for sensitivity, specificity, and accuracy) and CT ( $p<0.001$ for sensitivity and accuracy). PET/CT also correctly identified significantly more extra-nodal lesions (22) than CT (14) and PET (nine).

Conclusions: PET/CT provides significantly more accurate information compared to PET and CT for the staging and re-staging of patients with indolent lymphoma.
\end{abstract}

Key words: PET, PET/CT, Indolent lymphoma

\section{Introduction}

$\mathrm{M}$ etabolic imaging with fluorine-18-fluorodeoxyglucose (FDG) positron emission tomography (PET) has become the clinical standard for staging and evaluation of treatment response in patients with non-Hodgkin lymphoma (NHL) [1-5]. More recently, PET/computed tomography (CT) has been shown to stage lymphoma with a higher accuracy than PET alone [6-9]. These studies have focused on Hodgkin's disease or aggressive NHL.

Staging of disease has important treatment implications for indolent NHL (indolent lymphoma). For

Correspondence to: Martin S. Allen-Auerbach; e-mail: mauerbach@ mednet.ucla.edu example, up to one third of patients with follicular lymphoma can present with stage I and II disease, and $70 \%$ to $90 \%$ of mucosa-associated lymphoid tissue (MALT) lymphomas may present with localized (stage IE-IIE) disease [10]. In these cases, patients can be treated with radiation only [11], whereas higher stages usually require systemic therapy.

However, very few studies have evaluated the role of FDG PET in patients with indolent lymphoma. Most of these studies concluded that PET appears useful for the staging of the follicular subtype but only has a limited usefulness in the other subtypes of indolent lymphoma [12-15]. Except for a small study in patients with MALT lymphoma by Perry et al. [16], no study has evaluated the role of PET/CT in the staging of indolent lymphoma. 
FDG uptake in indolent lymphoma appears to be lower than in aggressive lymphomas [17]. Data on the correlation between proliferative activity and glycolysis in malignant tissue, as measured by FDG uptake, are controversial [18, 19]. To date, the correlation between FDG uptake and proliferative activity specifically in indolent lymphoma has not been studied in detail.

To our knowledge, to date, no study has investigated the value of PET/CT over PET and CT for staging of indolent lymphoma. The aim of the present study was to investigate the potential impact of PET/CT as compared to PET and CT on the staging of patients with indolent lymphoma. For a better understanding of FDG uptake in indolent lymphoma, we also investigated the correlation between FDG uptake and proliferative activity as measured by Ki-67 staining.

\section{Patients and Methods}

\section{Patients}

From March 2004 to September 2006, 301 patients with indolent lymphoma were referred to our institution for PET/CT imaging. In 45 of these patients (26 male, 19 female, mean age of $56 \pm 13$ years) referred for staging $(n=20)$ or restaging $(n=25)$, a reliable reference standard was available. The reference standard was established by clinical follow-up including additional imaging in all patients and additional biopsy in 26 patients.

The histological tumor type was follicular lymphoma in $47 \%$ $(n=21)$ of patients, nodal and extranodal marginal zone lymphoma in $26 \%(n=12)$, small lymphocytic lymphoma in $9 \%(n=4)$, mantle cell lymphoma in $9 \%(n=4)$ as well as lymphoplasmocytic lymphoma $(n=1)$, cutaneous T-cell lymphoma $(n=2)$, and T-cell/ NK lymphoproliferative disorder $(n=1)$ in altogether $9 \%$ (see Table 1).

\section{Image Acquisition}

PET/CT was performed using the Reveal RT scanner (Siemens Medical Systems). This system combines a dual-slice-detector CT scanner (Somatom Emotion) with an ECAT ACCEL PET scanner. The characteristics of the device have been described in detail previously [20].

Table 1. Patient characteristics

\begin{tabular}{ll}
\hline $\begin{array}{l}\text { Number of patients } \\
\text { Gender }\end{array}$ & 45 \\
Female & 19 \\
Male & 26 \\
Age (years) & \\
Mean & 56 \\
Range & $21-78$ \\
Histology & $21(47 \%)$ \\
Follicular & $12(26 \%)$ \\
Marginal Zone (including MALT) & $4(9 \%)$ \\
Small lymphocytic lymphoma & $4(9 \%)$ \\
Mantle cell lymphoma & $4(9 \%)$ \\
Others (lymphoplasmocytic, cutaneous T-cell, T-cell/ NK) & \\
Scan Indication & 20 \\
Initial staging & 25 \\
Restaging & \\
\hline
\end{tabular}

While resting on a reclining chair, the patients received a $7.77 \mathrm{MBq}(0.21 \mathrm{mCi})$ per kg bodyweight of ${ }^{18} \mathrm{~F}-\mathrm{FDG}$ intravenously (up to a maximum of $740 \mathrm{MBq}$ [20 mCi]) and were asked to drink $900 \mathrm{~mL}$ of barium sulfate. The imaging sequence began $45 \mathrm{~min}$ after tracer injection. All patients were positioned on the imaging table with their arms up. After determining the imaging field (base of skull to mid thighs) with an initial scout scan, CT acquisition with intravenous contrast material was performed using the following parameters: $130 \mathrm{kVp}, 120 \mathrm{mAs}$, 1-s tube rotation, 4$\mathrm{mm}$ slice collimation, and bed speed of $8 \mathrm{~mm} / \mathrm{s}$ (i.e., pitch of 2).

The CT scan was followed by the PET emission scan, using a weight-based protocol as recently described [20, 21]. Scanning encompassed six to eight bed positions (depending on patient height) from the mid-thigh to the base of the skull. PET images were acquired in 3D mode. Patients were instructed to breathe shallowly during the PET and CT portions of the study to minimize misregistration between PET and CT images [22].

\section{Image Reconstruction}

CT images were reconstructed using conventional filtered backprojection, at 3.4-mm axial intervals to match the slice separation of the PET data. PET images were reconstructed by using iterative algorithms (ordered-subsets expectation maximization, two iterations and eight subsets) to a final image resolution of $8.8 \mathrm{~mm}$ at full width at half maximum. Attenuation was corrected by mapping the CT Hounsfield units to the linear attenuation coefficients as previously described [23].

\section{Image Analysis}

$\mathrm{PET}, \mathrm{CT}$, and PET/CT acquired in this retrospectively enrolled population were interpreted prospectively as follows: CT and PET images were interpreted independently by one radiologist and one nuclear medicine specialist, respectively, who were blinded to any additional clinical and other imaging findings. PET/CT studies were read in consensus.

A lesion was classified as positive on PET if there was focally increased FDG uptake above background that was not explained by physiological activity. A lesion on CT was classified by lymph node size criteria (diameter $>10 \mathrm{~mm}$ ) or extra-nodal structural anatomical abnormality.

Unlike aggressive disease, indolent lymphomas do not exhibit uniformly increased FDG uptake [12-15]. Therefore, our image interpretation criteria were biased toward CT as follows: on PET/ $\mathrm{CT}$, a lymph node was classified as abnormal if there was increased FDG uptake and/or abnormal size regardless of FDG uptake. Extranodal lesions were interpreted as abnormal if they exhibited focally increased FDG uptake and/or pathological anatomical features on CT (regardless of the FDG uptake).

\section{Staging and Analysis of Nodal Regions}

Based on the lymph node regions originally defined by Kaplan and Rosenberg[24], we analyzed 13 lymph node regions per patients. Therefore, a total of 585 lymph node regions were analyzed. The patient stage was determined based on the Ann Arbor classification [25] from the number of positive nodal and extra-nodal regions by $\mathrm{PET}, \mathrm{CT}$, and PET/CT. 


\section{Quantitative Analysis of FDG Uptake}

For the calculation of SUV, circular regions of interest (15-mm diameter) were placed on consecutive axial images of lesions visually identified to have abnormally increased FDG uptake. In the absence of visual FDG uptake, the biopsy site was used for the determination of SUV. The SUV was calculated as:

$\mathrm{SUV}=\frac{\text { decay }- \text { corrected activity }(\mathrm{kBq}) / \text { tissue volume }(\mathrm{ml})}{\text { injected }- \text { FDG activity }(\mathrm{kBq}) / \text { body weight }(\mathrm{g})}$

To minimize partial volume effects and assure reproducibility of measurements, the maximum SUV $\left(\mathrm{SUV}_{\max }\right)$ was used.

\section{Immunohistochemical Staining with Ki-67}

Ki-67 staining results from biopsies obtained for diagnostic purposes were available in 29 patients who had not undergone treatment between the biopsy and the PET/CT. Biopsies were performed within $4.38 \pm 4.79$ weeks $(0-16.43)$ of the PET/CT. The Ki-67 labeling index reflects cell proliferative activity and is defined as the percentage of nuclei stained per total number of nuclei in the sample. The immunohistochemistry and determination of the Ki-67 labeling index were performed according to standard protocol and routine at our institution [26, 27].

Proliferative activity by Ki-67 staining was then correlated with the $\mathrm{SUV}_{\max }$ of the biopsied site.

\section{SUV $V_{\text {max }}$ and Histology}

$\mathrm{SUV}_{\text {max }}$ was also correlated with histology to determine whether the type of indolent lymphoma determines FDG uptake.

\section{Standard of Reference}

All patients had lymph node biopsy for histological diagnosis of disease. For ethical reasons, systematic biopsy of all sites for staging was not performed. Clinical evaluation and follow-up for $71 \pm 32$ weeks (range, 16 to 131 weeks) for all patients, biopsy $(n=26)$, and additional imaging consisting of CT, MRI or PET/CT $(n=35)$ performed $4.55 \pm$ 4.25 weeks after the initial scan (range, 0 to 17 weeks) served as the reference standard. Concordant positive findings of clinical evaluation, $\mathrm{CT}$, PET, and PET/CT were regarded as true sites of disease. Concordant negative findings of clinical evaluation, CT, PET, and PET/CT were regarded as true absence of diseases. In cases of discordance between PET, CT, and PET/CT, response to treatment and follow-up data were used to assess the overall accuracy of the patient's disease status. Lesions were considered true positive if abnormalities either persisted on a follow-up PET or CT scan with no interval treatment or resolved on a follow-up scan in patients that had received interval treatment. Conversely, lesions that resolved on follow-up scanning without interval treatment were considered false positive.

\section{Statistics}

Using the standard of reference, sensitivity, specificity, and accuracy were calculated [28]. In addition, comparison between groups was performed using the unpaired $t$ test and McNemar test. Correlations were sought using the Pearson correlation. A $p<0.05$ was considered significant.

\section{Results}

Thirty-three of 45 patients had evidence of active disease with a total of 117 of 585 nodal regions involved according to the reference standard. In addition, a total of 36 extranodal lesions were identified by the reference standard.

\section{Staging Performance}

Compared to the reference standard, the correct stage was determined by PET in $62 \%$ of patients $(n=28)$, by $\mathrm{CT}$ in $58 \% \quad(n=26)$, and by PET/CT in $76 \% \quad(n=34)$. PET incorrectly assigned a higher stage in $7 \%$ of patients $(n=3)$, $\mathrm{CT}$ in $7 \%(n=3)$, and PET/CT in $9 \%(n=4)$. PET incorrectly assigned a lower stage in 31\% $(n=14)$, CT in $36 \%(n=16)$, and $\mathrm{PET} / \mathrm{CT}$ in $16 \%(n=7)$. There was no significant difference between the number of patients correctly staged by PET and PET/CT $(p=0.24)$, and PET/CT only demonstrated a trend toward better performance than $\mathrm{CT}(p=0.079)$.

In detail, PET/CT correctly resulted in a higher stage than $\mathrm{CT}$ in $18 \%(n=8)$ of the patients and in a higher stage than PET in $20 \%(n=9)$ of patients. PET/CT correctly downstaged $4 \%(n=2)$ of patients compared to PET. Due to the selected image interpretation criteria, down-staging of CT by $\mathrm{PET} / \mathrm{CT}$ was not possible.

\section{Positive Nodal and Extra-nodal Regions}

From a total of 585 nodal regions, PET/CT correctly diagnosed 92 nodal regions as positive for lymphomatous involvement and 458 as disease free vs 68 and 449 for PET and 64 and 459 for CT, respectively. The number of falsepositive and false-negative nodal regions were eight and 27 for PET/CT, 18 and 50 for PET, and eight and 54 for CT, respectively. The respective sensitivities, specificities, and accuracies were $99 \%, 100 \%$, and $99.8 \%$ for PET/CT, $68 \%$, $97.5 \%$, and $92.2 \%$ for PET, and $70 \%, 100 \%$, and $94.7 \%$ for CT. PET/CT performed significantly better than PET $(p<$ 0.001 for sensitivity, specificity, and accuracy) and CT ( $p<$ 0.001 for sensitivity and accuracy). Differences between PET and CT were not significant for sensitivity, significant for specificity $(p<0.001)$, and tended to be significant for accuracy ( $p=0.089$; see Table 2 ).

$\mathrm{PET} / \mathrm{CT}$ was also significantly better in detecting extra-nodal lesions than CT $(p<0.016)$ and PET $(p=$ 0.06 ). Of the 36 extranodal lesions by gold standard (tissues /organs involved were the parotid gland, lung, stomach, liver, adrenal gland, kidney, spleen, skin, conjunctiva, meninges, the bone marrow, as well as muscle and soft tissue), PET/CT detected 25 extra-nodal lesions, CT 16, and PET nine (see Figs. 1 and 2). Of eight bone marrow sites, three were identified by PET, four by PET/CT, and none by CT alone. Of eight sites of lung involvement, PET only noted three, whereas CT and PET/CT correctly diagnosed all eight. From nine sites of soft tissue involvement, PET noted one, CT four, 
Table 2. Performance for detection of nodal disease

\begin{tabular}{llll}
\hline & Sensitivity & Specificity & Accuracy \\
\hline PET & 68 & 97.5 & 92.2 \\
CT & 70 & 100 & 94.7 \\
PET/CT & 99 & 100 & 99.8 \\
& $p<0.001$ vs PET, CT & $p<0.001$ vs PET & $p<0.001$ vs PET, CT \\
\hline
\end{tabular}

and PET/CT eight. Of 12 sites with organ involvement, PET correctly noted two, CT four, and PET/CT five.

The above includes six patients with extranodal marginal zone lymphoma. One of these six patients did not have active disease. Of the five remaining patients, organ involvement included the meninges, the parotid gland, conjunctiva, lung, liver, stomach, spleen, kidney, and the skin.

\section{$S U V_{\max }$ vs Proliferative Activity and Histology}

$\mathrm{SUV}_{\max }$ ranged from 0.4 to 15.4 (mean $\left.\pm \mathrm{SD}, 3.7 \pm 4.1\right)$ in follicular and from 0.8 to $9.8(2.2 \pm 2.1)$ in non-follicular indolent lymphomas. The highest $\mathrm{SUV}_{\max }$ as well as the lowest $\mathrm{SUV}_{\max }$ occurred in follicular grade-1 lymphomas.

There was also a wide range of $\mathrm{Ki}-67$ staining with the lowest proliferative activity observed in MALT lymphoma and the highest in mantle cell lymphoma. The correlation of $\mathrm{SUV}_{\max }$ and Ki-67 index was not statistically significant.

No significant difference was found between the $\mathrm{SUV}_{\max }$ of the various types of indolent lymphoma.

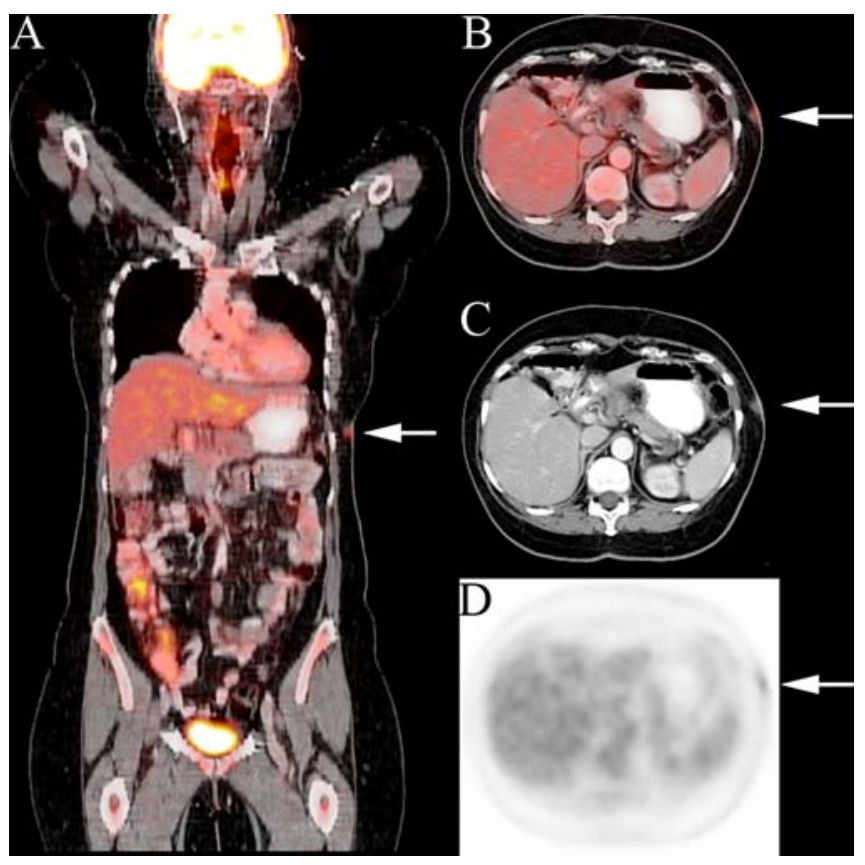

Fig. 1. A 59-year-old woman with a history of cutaneous Tcell lymphoma. Fused coronal (a) and axial PET/CT (b) slices demonstrate left flank skin involvement (arrow) missed on CT (c) and PET (d) interpretation.

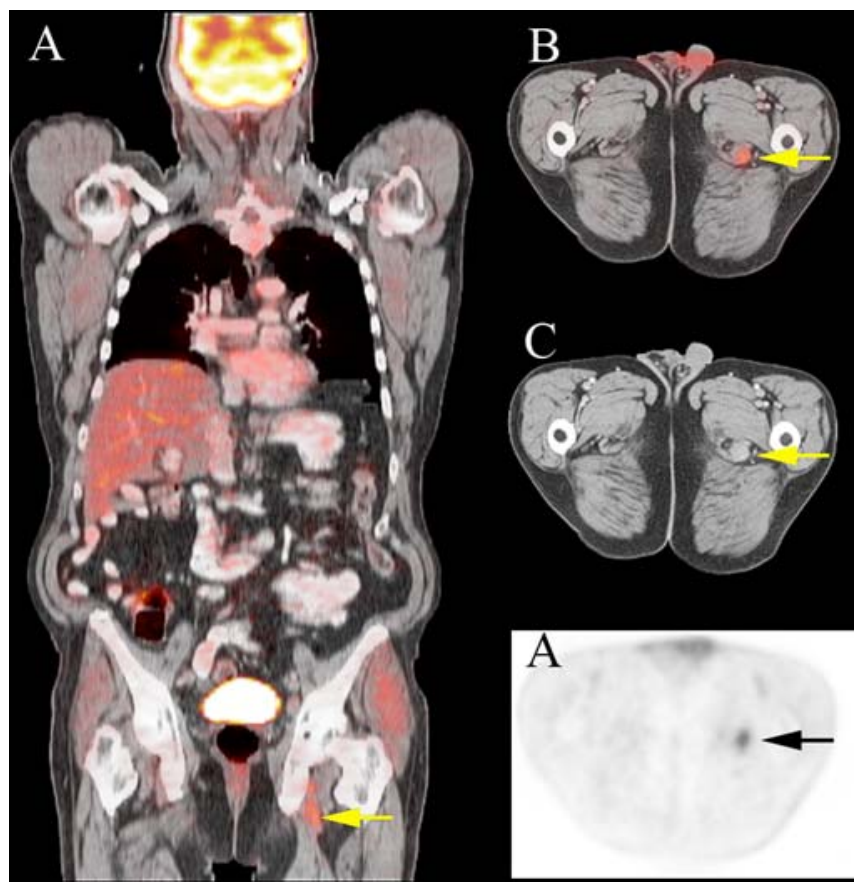

Fig. 2. A 62-year-old man with history of mantle cell lymphoma. Fused coronal (a) and axial PET/CT (b) slices demonstrate disease related FDG uptake in left obturator adenopathy (arrow) missed on CT (c) and PET (d) interpretation.

\section{Discussion}

For the group of patients studied, PET/CT was able to add clinically important information not available from CT or PET alone. On a regional-based analysis of patients with indolent lymphoma, PET/CT performed significantly better than PET and CT in correctly classifying lymph node groups as positive or negative for lymphomatous involvement. PET/ CT was also able to detect more extra-nodal sites of disease than either CT or PET. There was no significant difference between the number of patients correctly staged by PET and $\mathrm{PET} / \mathrm{CT}$, and PET/CT only demonstrated a trend toward better performance than $\mathrm{CT}(p=0.079)$.

Few papers have looked at the value of FDG-PET over conventional imaging specifically for the staging of indolent lymphoma. Jerusalem et al. [13] reported that, in 42 patients with indolent lymphoma, PET was able to identify more lymph node areas infiltrated by indolent lymphoma compared to conventional staging procedures. Contrary to the findings of Jerusalem et al., we found no significant difference between the sensitivity of PET or CT for the detection of nodal groups with disease. However, PET/CT was significantly better in detecting nodal disease than either modality. Similarly, PET/CT detected more extranodal disease sites than PET or CT. For the overall staging of disease, Jerusalem et al. reported that PET plus a bone marrow biopsy compared to conventional staging procedures (also including a bone marrow biopsy) observed the same stage in 37 patients, a higher stage by PET in two patients, 
and a higher stage by conventional procedures in three patients. In our study, PET/CT staged more patients correctly than either PET or CT, although the differences for overall staging only demonstrated a statistical trend to significance. Najjar et al. [29] reported a similar observation finding the combined information of separately acquired CT and PET more sensitive for staging indolent lymphoma than either modality alone. Disease occurring in normal appearing lymph nodes or extra-nodal sites can be picked up by PET. Conversely, CT can detect disease missed by PET either because of size or because of the absence of FDG uptake frequently encountered in the setting of indolent lymphoma. The additional value of fused imaging with PET/CT over side by side reading of CT and PET has been reported for multiple malignancies in general [30] as well as specifically for lymphoma $[6,7]$.

Data on the correlation between proliferative activity determined by Ki-67 staining and glucose metabolism in lymphomas, as measured by FDG uptake, are controversial. Okada et al. [18] report a positive correlation between proliferation and FDG uptake in a small series of patients with malignant lymphomas. We observed no statistically significant correlation between $\mathrm{SUV}_{\max }$ and percentage of cells that stained positive with Ki-67. We therefore feel that, in indolent lymphoma, $\mathrm{SUV}_{\max }$ cannot substitute for the prognostic value provided by $\mathrm{Ki}-67$ reported in indolent lymphoma [31].

Comparing the $\mathrm{SUV}_{\max }$ of the different subgroups of indolent lymphoma in our study, we found no significant differences in $\mathrm{SUV}_{\max }$. Although Karam et al. [12] did note a significantly higher SUV in follicular lymphoma than other indolent lymphoma, our findings are more in line with Schoeder et al. [17], noting no significant difference in FDG uptake within the histological subtypes of indolent lymphoma. Thus, it is likely that histological classification based on SUV measurements cannot be a substitute for the histological diagnosis based on actual tissue. However, as BodetMilin et al. reported [32], PET/CT might be of value in the assessment of patients with indolent lymphoma suspected to have undergone histological transformation of their indolent lymphomas, since this usually results in a significant increase of FDG uptake at the sites of transformation.

\section{Limitations}

Since biopsy of every suspicious lesion would not be ethical, a surrogate gold standard had to be found. Concordant positive findings of clinical evaluation, CT, PET, and PET/CT were regarded as true sites of disease. Concordant negative findings of clinical evaluation, CT, PET, and PET/CT were regarded as true absence of disease. In cases of discordance between PET, $\mathrm{CT}$, and $\mathrm{PET} / \mathrm{CT}$, response to treatment and follow-up data were used to assess the overall accuracy of the patient's disease status. Lesions were considered true positive if abnormalities either persisted on a follow-up PET or CT scan with no interval treatment or resolved on a follow-up scan in patients that had received interval treatment. Conversely, lesions that resolved on follow-up scanning without interval treatment were considered false positive. Since initial treatment response to indolent lymphoma is excellent [33-35], lesions that would have persisted despite interval treatment would also have been considered false positive. However, we did not encounter any patient in which this would have been the case.

The patient population studied was heterogeneous, and larger studies evaluating the individual subtypes of indolent lymphomas might be warranted to confirm our results.

The relatively low number of patients with a reliable reference standard (45 out of 301) likely results from the clinical course low grade lymphomas frequently take, i.e., long periods of clinically inactive disease only leading to a clinical evaluation by the treating physician if symptoms occur.

\section{Conclusion}

PET/CT provides important additional information over PET and $\mathrm{CT}$ for the staging and re-staging of patients with indolent lymphoma. Thus, the application of PET/CT rather than CT or PET is likely to be of benefit in the management of patients with indolent lymphoma. Given the frequent absence of significant FDG uptake in indolent lymphomas, in the untreated patient, enlarged nodes detected on CT should be regarded as pathological even in the absence of FDG uptake. On subsequent post-treatment scans, node size should be used to evaluate for treatment response rather than FDG uptake.

Open Access. This article is distributed under the terms of the Creative Commons Attribution Noncommercial License which permits any noncommercial use, distribution, and reproduction in any medium, provided the original author(s) and source are credited.

\section{References}

1. Hoh CK, Glaspy J, Rosen P et al (1997) Whole-body FDG-PET imaging for staging of Hodgkin's disease and lymphoma. J Nucl Med 38(3):343-348

2. Jerusalem G, Beguin Y, Fasotte M et al (1999) Whole body positron emission tomograpohy using $18 \mathrm{~F}$-fluorodeoxyglucose for posttreatment evaluation in Hodgkin's disease and non-Hodgkin's lymphoma has higher diagnostic and prognostic value than classical computed tomography scan imaging. Blood 94:429-433

3. Römer W, Hanauske A, Ziegler S et al (1998) Positron emission tomography in non-Hodgkin's lymphoma: assessment of chemotherapy with fluorodeoxyglucose. Blood 91:4464-4471

4. Buchmann I, Reinhardt M, Elsner K et al (2001) 2-(Fluorine-18)fluoro2-deoxy-D-glucose positron emission tomography in the detection and staging of malignant lymphoma. A bicenter trial. Cancer 91(5):889-899

5. Reinhardt MJ, Herkel C, Altehoefer C, Finke J, Moser E (2005) Computed tomography and $18 \mathrm{~F}-\mathrm{FDG}$ positron emission tomography for therapy control of Hodgkin's and non-Hodgkin's lymphoma patients: when do we really need FDG-PET? Ann Oncol 16(9):1524-1529

6. Freudenberg LS, Antoch G, Schutt P et al (2004) FDG-PET/CT in restaging of patients with lymphoma. Eur J Nucl Med Mol Imaging 31 (3):325-329

7. Allen-Auerbach M, Quon A, Weber WA et al (2004) Comparison between 2-deoxy-2-[18F]fluoro-D-Glucose positron emission tomography and positron emission tomography/computed tomography hardware fusion for staging of patients with lymphoma. Mol Imaging Biol 6(6):411-416 
8. Hernandez-Maraver D, Hernandez-Navarro F, Gomez-Leon $\mathrm{N}$ et al (2006) Positron emission tomography/computed tomography: diagnostic accuracy in lymphoma. Br J Haematol 135(3):293-302

9. Hutchings M, Loft A, Hansen M et al (2006) Position emission tomography with or without computed tomography in the primary staging of Hodgkin's lymphoma. Haematologica 91(4):482-489

10. Armitage JO, Weisenburger DD (1998) New approach to classifying non-Hodgkin's lymphomas: clinical features of the major histologic subtypes. Non-Hodgkin's Lymphoma Classification Project. J Clin Oncol 16(8):2780-2795

11. Tsang RW, Gospodarowicz MK (2005) Radiation therapy for localized low-grade non-Hodgkin's lymphomas. Hematol Oncol 23(1):10-17

12. Karam M, Novak L, Cyriac J, Ali A, Nazeer T, Nugent F (2006) Role of fluorine-18 fluoro-deoxyglucose positron emission tomography scan in the evaluation and follow-up of patients with low-grade lymphomas. Cancer 107(1):175-183

13. Jerusalem G, Beguin Y, Najjar F et al (2001) Positron emission tomography (PET) with 18F-fluorodeoxyglucose (18F-FDG) for the staging of low-grade non-Hodgkin's lymphoma (NHL). Ann Oncol 12 (6): $825-830$

14. Jerusalem GH, Beguin YP (2002) Positron emission tomography in non-Hodgkin's lymphoma (NHL): relationship between tracer uptake and pathological findings, including preliminary experience in the staging of low-grade NHL. Clin Lymphoma 3(1):56-61

15. Blum RH, Seymour JF, Wirth A, MacManus M, Hicks RJ (2003) Frequent impact of [18F]fluorodeoxyglucose positron emission tomography on the staging and management of patients with indolent nonHodgkin's lymphoma. Clin Lymphoma 4(1):43-49

16. Perry C, Herishanu Y, Metzer U et al (2007) Diagnostic accuracy of $\mathrm{PET} / \mathrm{CT}$ in patients with extranodal marginal zone MALT lymphoma. Eur J Haematol 79(3):205-209

17. Schoder H, Noy A, Gonen M et al (2005) Intensity of 18 fluorodeoxyglucose uptake in positron emission tomography distinguishes between indolent and aggressive non-Hodgkin's lymphoma. J Clin Oncol 23(21):4643-4651

18. Okada J, Yoshikawa K, Itami M et al (1992) Positron emission tomography using fluorine-18-fluorodeoxyglucose in malignant lymphoma: a comparison with proliferative activity. J Nuc Med 33:325329

19. Higashi K, Clavo AC, Wahl RL (1993) Does FDG uptake measure proliferative activity of human cancer cells? In vitro comparison with DNA flow cytometry and tritiated thymidine uptake. J Nucl Med 34(3):414-419

20. Halpern BS, Dahlbom M, Quon A et al (2004) Impact of patient weight and emission scan duration on PET/CT image quality and lesion detectability. J Nucl Med 45(5):797-801
21. Halpern BS, Dahlbom M, Auerbach MA et al (2005) Optimizing imaging protocols for overweight and obese patients: a lutetium orthosilicate PET/CT study. J Nucl Med 46(4):603-607

22. Goerres GW, Burger C, Schwitter MR, Heidelberg TN, Seifert B, von Schulthess GK (2003) PET/CT of the abdomen: optimizing the patient breathing pattern. Eur Radiol 13(4):734-739

23. Kinahan PE, Townsend DW, Beyer T, Sashin D (1998) Attenuation correction for a combined 3D PET/CT scanner. Med Phys 25(10):2046-2053

24. Kaplan H, Rosenberg S (1996) The treatment of Hodgkin's disease. Med Clin North Am 50:1591

25. Carbone P, Kaplan H, Musshoff K, Smithers D, Tubiana M (1971) Report of the committee on Hodgkin's disease staging classification. Cancer Res 31:1860-1861

26. Sarantopoulos GP, Gui D, Shintaku P et al (2004) Immunohistochemical analysis of lung carcinomas with pure or partial bronchioloalveolar differentiation. Arch Pathol Lab Med 128(4):406-414

27. Terasaki H, Niki T, Matsuno Y et al (2003) Lung adenocarcinoma with mixed bronchioloalveolar and invasive components: clinicopathological features, subclassification by extent of invasive foci, and immunohistochemical characterization. Am J Surg Pathol 27(7):937-951

28. Bennett BM (1972) On comparisons of sensitivity, specificity and predictive value of a number of diagnostic procedures. Biometrics 28(3):793-800

29. Najjar F, Hustinx R, Jerusalem G, Fillet G, Rigo P (2001) Positron emission tomography (PET) for staging low-grade non-Hodgkin's lymphomas (NHL). Cancer Biother Radiopharm 16(4):297-304

30. Czernin J, Allen-Auerbach M, Schelbert HR (2007) Improvements in cancer staging with PET/CT: literature-based evidence as of September 2006. J Nucl Med 48(Suppl 1):78S-88S

31. Koster A, Tromp HA, Raemaekers JM et al (2007) The prognostic significance of the intra-follicular tumor cell proliferative rate in follicular lymphoma. Haematologica 92(2):184-190

32. Bodet-Milin C, Kraeber-Bodere F, Moreau P, Campion L, Dupas B, Le Gouill S (2008) Investigation of FDG-PET/CT imaging to guide biopsies in the detection of histological transformation of indolent lymphoma. Haematologica 93(3):471-472

33. Hainsworth JD (2003) First-line and maintenance treatment with rituximab for patients with indolent non-Hodgkin's lymphoma. Semin Oncol 30(1 Suppl 2):9-15

34. Witzig TE, Vukov AM, Habermann TM et al (2005) Rituximab therapy for patients with newly diagnosed, advanced-stage, follicular grade I non-Hodgkin's lymphoma: a phase II trial in the North Central Cancer Treatment Group. J Clin Oncol 23(6):1103-1108

35. Italiano A, Thyss A (2005) Follicular lymphoma: a therapeutic update. Bull Cancer 92(10):57-64 